Gordon Sinnamon, ${ }^{*}$ Department of Mathematics, University of Western Ontario, London, ONT N6A 5B7, Canada (email: sinnamon@@uwo.ca)

\title{
KUFNER'S CONJECTURE FOR HIGHER ORDER HARDY INEQUALITIES
}

\begin{abstract}
A conjecture of A. Kufner is verified, giving an approximate functional form of the Green's function for a class of two-point boundary value problems. The result is applied to give a simple characterization of those weights for which the Hardy inequality for higher order derivatives is valid.
\end{abstract}

\section{Introduction and Statement of Main Results}

The weighted Hardy inequality for derivatives of order $k \geq 1$ is

$$
\left(\int_{0}^{1}|u(x)|^{q} w_{0}(x) d x\right)^{1 / q} \leq C_{k}\left(\int_{0}^{1}\left|u^{(k)}(x)\right|^{p} w_{k}(x) d x\right)^{1 / p} .
$$

Here $k$ is a fixed positive integer, $w_{0}$ and $w_{k}$ are non-negative weight functions, $1<p<\infty$, and $0<q<\infty$.

Estimating the size of a function in terms of its derivatives is a problem of fundamental importance in real analysis and differential equations and the weighted Lebesgue spaces we consider arise naturally in such settings. The flexibility we have when applying the inequality (1) comes from the weight functions $w_{0}$ and $w_{k}$ so our object is to prove the inequality while placing the minimum restrictions on these weights. In this paper we give necessary and

Key Words: Hardy's inequality, Two-point Boundary Value Problem, Green's Function, Weighted Norm Inequality

Mathematical Reviews subject classification: Primary 26D10; Secondary 34B05, 46N20

Received by the editors July 13, 1995

* Support from the Natural Sciences and Engineering Research Council is gratefully acknowledged. 
sufficient conditions on $p, q, w_{0}$ and $w_{k}$ for there to exist a positive constant $C_{k}$ such that (1) holds for all functions $u$ from a certain class.

To describe the class of functions $u$ that we consider, let $N_{i}=\{0,1, \ldots, i-$ 1) and fix subsets $M_{0}$ and $M_{1}$ of $N_{k}$ such that $\left|M_{0}\right|+\left|M_{1}\right|=k$. We consider the class of all functions $u$ which are solutions to the BVP (boundary value problem)

$$
u^{(k)}=f ; \quad u^{(i)}(0)=0 \text { for } i \in M_{0}, \quad u^{(i)}(1)=0 \text { for } i \in M_{1}
$$

for some locally integrable function $f$.

The validity of (1) over this class of functions $u$ depends strongly on the pair of sets $\left(M_{0}, M_{1}\right)$. Drábek and Kufner [3] have shown that the inequality is meaningful if and only if $\left(M_{0}, M_{1}\right)$ satisfies the Pólya condition:

$$
\left|M_{0} \cap N_{i}\right|+\left|M_{1} \cap N_{i}\right| \geq i, \quad i=1,2, \ldots, k .
$$

To better understand this condition we introduce the $2 \times k$ incidence matrix $E=\left(e_{\alpha i}\right)$ of $\left(M_{0}, M_{1}\right)$ by setting $e_{\alpha i}=1$ if $i-1 \in M_{\alpha}$ and $e_{\alpha i}=0$ otherwise. The condition (3) states that there are at least $i$ 1's in the first $i$ columns of $E$ for $i=1,2, \ldots, k$.

For a pair $\left(M_{0}, M_{1}\right)$ satisfying the Pólya condition (3), it is proved in [3] that the BVP (2) can be uniquely solved. There is, therefore, a Green's function $G(x, t)$ for the BVP (see, for example, [2, p162 ff]) so that for any locally integrable function $f$, the solution of (2) is given by

$$
u(x)=\int_{0}^{1} G(x, t) f(t) d t
$$

Moreover, $G$ has the form

$$
G(x, t)=\frac{(x-t)^{k-1}}{(k-1) !} \chi_{(0, x)}(t)+\sum_{i=0}^{k-1} \sum_{j=0}^{k-1} w_{i j} \frac{x^{i}}{i !} \frac{t^{j}}{j !}
$$

for some constants $w_{i j}$ depending on $\left(M_{0}, M_{1}\right)$. Here and in the sequel, $\chi_{S}$ denotes the function that takes the value 1 on the set $S$ and 0 elsewhere.

The connection between the Green's function $G(x, t)$ and the weight characterization for the inequality (1) is a conjecture of A. Kufner [4] and [5] which describes an approximation to $G(x, t)$ and a weight characterization for (1) based on that approximation. It is the purpose of this paper to verify the conjecture and thereby complete the proof of the weight characterization.

Before stating the conjecture we consider some special cases and make a convenient definition. 
If $k=1,(1)$ reduces to the weighted Hardy inequality characterized in [9], [1], [8], [11] and elsewhere. (See [10] for proofs and further references.) We will assume that $k \geq 2$ henceforth.

If all boundary conditions are at one endpoint, that is, if

$$
\left(M_{0}, M_{1}\right)=\left(N_{k},\{\}\right) \text { or }\left(M_{0}, M_{1}\right)=\left(\{\}, N_{k}\right),
$$

then again inequality (1) has been characterized. See [12] and references therein. We will not consider these cases here.

Other special cases have been solved in [6], [7], [5].

Definition 1.1 For a pair $\left(M_{0}, M_{1}\right)$ satisfying (3) but not (5) we define nonnegative integers $a, b, c$, and $d$, as follows: Let a be the number of consecutive 1 's beginning the top row of $E ; b$ be the number of consecutive 1's beginning the bottom row of $E$; $c$ be the number of consecutive 0's ending the top row of $E$; and $d$ be the number of consecutive 0's ending the bottom row of $E$.

To illustrate the definition we offer an example. Take $k=6, M_{0}=$ $\{0,1,2,4\}$, and $M_{1}=\{1,3\}$. Then we have

$$
E=\left(\begin{array}{llllll}
1 & 1 & 1 & 0 & 1 & 0 \\
0 & 1 & 0 & 1 & 0 & 0
\end{array}\right)
$$

$a=3, b=0, c=1$, and $d=2$. Notice that $\left(M_{0}, M_{1}\right)$ satisfies the Pólya condition.

For any $\left(M_{0}, M_{1}\right), a+c \leq k$ and $b+d \leq k$. Notice that $a+c=k-1$ is impossible, this will be important later.

We state Kufner's conjecture in a somewhat different form than it appears in $[5]$.

Theorem 1.2 Suppose $\left(M_{0}, M_{1}\right)$ satisfies $\left|M_{0}\right|+\left|M_{1}\right|=k$ and (3) but not (5). The Green's function, $G(x, t)$, of the BVP (2) satisfies

$$
c_{1}|G(x, t)| \leq x^{a}(1-x)^{B} t^{C}(1-t)^{d} \leq c_{2}|G(x, t)|, \text { for } 0<x<t<1,
$$

and

$$
c_{1}|G(x, t)| \leq x^{A}(1-x)^{b} t^{c}(1-t)^{D} \leq c_{2}|G(x, t)|, \text { for } 0<t<x<1,
$$

for some positive constants $c_{1}$ and $c_{2}$. Here $A, B, C$, and $D$ are given by

$$
A=\left\{\begin{array}{ll}
a-1, & \text { if } a+c=k \\
a, & \text { if } a+c<k
\end{array}, \quad C=\left\{\begin{array}{ll}
c-1, & \text { if } a+c=k \\
c, & \text { if } a+c<k
\end{array},\right.\right.
$$




$$
B=\left\{\begin{array}{ll}
b-1, & \text { if } b+d=k \\
b, & \text { if } b+d<k
\end{array}, \quad D=\left\{\begin{array}{ll}
d-1, & \text { if } b+d=k \\
d, & \text { if } b+d<k
\end{array} .\right.\right.
$$

Before proving Theorem 1.2 we show how approximating the Green's function gives necessary and sufficient conditions for inequality (1). The next result follows from Theorem 1.2 above and Corollary 1.5 in [5].

Theorem 1.3 Let $k \geq 2$ and suppose $\left(M_{0}, M_{1}\right)$ satisfies $\left|M_{0}\right|+\left|M_{1}\right|=k$ and (3) but not (5). Define $p^{\prime}$ and $r$ by $1 / p+1 / p^{\prime}=1$ and $1 / r=1 / q-1 / p$ and consider the statements (i), (ii), and (iii) below. If $1<p \leq q<\infty$, then (i) holds if and only if (ii) holds. If $0<q<p<\infty$ and $1<p$, then (i) holds if and only if (iii) holds.

(i) There is a positive constant $C_{k}$ such that

$$
\left(\int_{0}^{1}|u(x)|^{q} w_{0}(x) d x\right)^{1 / q} \leq C_{k}\left(\int_{0}^{1}\left|u^{(k)}(x)\right|^{p} w_{k}(x) d x\right)^{1 / p} .
$$

holds for all $u$ whose derivatives of order $k-1$ are absolutely continuous on $[0,1]$ and which satisfies $u^{(i)}(0)=0$ for $i \in M_{0}$ and $u^{(i)}(1)=0$ for $i \in M_{1}$.

(ii) The following two suprema are finite.

$$
\begin{aligned}
& \sup _{0<s<1}\left[\int_{s}^{1} x^{A q}(1-x)^{b q} w_{0}(x) d x\right]^{1 / q}\left[\int_{0}^{s} t^{c p^{\prime}}(1-t)^{D p^{\prime}} w_{k}(t)^{1-p^{\prime}} d t\right]^{1 / p^{\prime}} . \\
& \sup _{0<s<1}\left[\int_{0}^{s} x^{a q}(1-x)^{B q} w_{0}(x) d x\right]^{1 / q}\left[\int_{s}^{1} t^{C p^{\prime}}(1-t)^{d p^{\prime}} w_{k}(t)^{1-p^{\prime}} d t\right]^{1 / p^{\prime}} .
\end{aligned}
$$

(iii) The following two integrals are finite.

$$
\begin{aligned}
& \int_{0}^{1}\left[\int_{s}^{1} x^{A q}(1-x)^{b q} w_{0}(x) d x\right]^{r / p} \times \\
& \times\left[\int_{0}^{s} t^{c p^{\prime}}(1-t)^{D p^{\prime}} w_{k}(t)^{1-p^{\prime}} d t\right]^{r / p^{\prime}} s^{A q}(1-s)^{b q} w_{0}(s) d s . \\
& \int_{0}^{1}\left[\int_{0}^{s} x^{a q}(1-x)^{B q} w_{0}(x) d x\right]^{r / p} \times \\
& \times\left[\int_{s}^{1} t^{C p^{\prime}}(1-t)^{d p^{\prime}} w_{k}(t)^{1-p^{\prime}} d t\right]^{r / p^{\prime}} s^{a q}(1-s)^{B q} w_{0}(s) d s .
\end{aligned}
$$

Here $a, b, c, d, A, B, C$, and $D$ depend on $\left(M_{0}, M_{1}\right)$ as in Definition 1.1 and Theorem 1.2. 
The weight characterization simplifies if both $a+c<k$ and $b+d<$ $k$ because in this case $G(x, t)$ can be approximated by the function $x^{a}(1-$ $x)^{b} t^{c}(1-t)^{d}$ on the whole unit square. We state this precisely below.

Theorem 1.4 Let $k \geq 2$ and suppose that $\left(M_{0}, M_{1}\right)$ satisfies $\left|M_{0}\right|+\left|M_{1}\right|=k$ and (3) but not (5). Also suppose that $a+c<k$ and $b+d<k$. Then statement (i) of Theorem 1.2 holds if and only if

$$
\left[\int_{0}^{1} x^{a q}(1-x)^{b q} w_{0}(x) d x\right]^{1 / q}\left[\int_{0}^{1} t^{c p^{\prime}}(1-t)^{d p^{\prime}} w_{k}(t)^{1-p^{\prime}} d t\right]^{1 / p^{\prime}}<\infty .
$$

Here $0<q<\infty, 1<p<\infty$, and $1 / p+1 / p^{\prime}=1$.

Proof. If we write $u^{(k)}=f$, then $u(x)=\int_{0}^{1} G(x, t) f(t) d t$ and (1) becomes

$$
\left(\int_{0}^{1}\left|\int_{0}^{1} G(x, t) f(t) d t\right|^{q} w_{0}(x) d x\right)^{1 / q} \leq C_{k}\left(\int_{0}^{1}|f(t)|^{p} w_{k}(t) d t\right)^{1 / p} .
$$

By Theorem 1.2, $G(x, t)$ does not change sign so we may restrict our attention to non-negative functions $f$. In this case the approximation for $G$ from Theorem 1.2 shows that the above inequality is equivalent to

$$
\left[\int_{0}^{1} x^{a q}(1-x)^{b q} w_{0}(x) d x\right]_{0}^{1 / q} \int_{0}^{1} t^{c}(1-t)^{d} f(t) d t \leq C_{k}^{\prime}\left(\int_{0}^{1} f(t)^{p} w_{k}(t) d t\right)^{1 / p}
$$

for some constant $C_{k}^{\prime}$. Taking the supremum over all non-negative functions $f$ and using the sharpness of Hölder's inequality we obtain the conclusion of the theorem.

\section{Approximating the Green's Function}

For the remainder of the paper we will regard $M_{0}$ and $M_{1}$ as fixed subsets of $N_{k}$ satisfying $\left|M_{0}\right|+\left|M_{1}\right|=k$ and (3) but not (5). This implies that the Green's function $G$ and the coefficients $w_{i j}$ from (4) are also fixed.

We begin with an argument that counts the zeros of $G$ and its partial derivatives.

Proposition 2.1 Let $t$ be in $(0,1)$ and set $g(x)=G(x, t)$. Then for $0 \leq i \leq$ $k-1$,

(a) $g(x) \neq 0$ for $x \in(0,1)$,

(b) $g^{(i)}(0)=0$ if and only if $i \in M_{0}$, and

(c) $g^{(i)}(1)=0$ if and only if $i \in M_{1}$. 
Proof. From the formula (4) it is clear that $g$ has $k-2$ continuous derivatives and that $g^{(k-2)}$ is not differentiable. In particular, none of $g, g^{\prime}, \ldots, g^{(k-2)}$ is identically zero. Thus if we let $S_{i}$ be the (closed) support of $g^{(i)}$, then $S_{i}$ is not empty. Since $g$ is a polynomial on $[0, t]$ and on $[t, 1], S_{i}$ is one of $[0,1]$, $[0, t]$, or $[t, 1]$.

Let $z_{i}$ be the number of zeros of $g^{(i)}$ in $S_{i}$. Take $L_{i}$ to be 1 if $g^{(i)}$ has a zero at the left endpoint of $S_{i}$ and 0 otherwise. Take $R_{i}$ to be 1 if $g^{(i)}$ has a zero at the right endpoint of $S_{i}$ and 0 otherwise.

We have the trivial inequality

$$
z_{0} \geq L_{0}+R_{0}
$$

If $0<i \leq k-2$, then $g^{(i-1)}$ is constant off $S_{i}$ so the $z_{i-1}$ zeros of $g^{(i-1)}$ that lie in $S_{i-1}$ are, in fact, in $S_{i}$. By the Mean Value Theorem, $g^{(i)}$ has at least $z_{i-1}-1$ zeros in the interior of $S_{i}$. Thus

$$
z_{i} \geq z_{i-1}-1+L_{i}+R_{i}, \quad 0<i \leq k-2 .
$$

If $L_{i}=0$, then either $g^{(i)}(0) \neq 0$ or $S_{i}=[t, 1]$ and $g^{(i)}(t) \neq 0$. The latter is impossible since $g^{(i)}$ is continuous. Since $g$ is a section of the Green's function of the BVP (2), $g$ must satisfy the boundary conditions, (see, for example, $[2$, p165]) so the former implies that $i \notin M_{0}$. Thus

$$
L_{i} \geq \chi_{M_{0}}(i), \quad 0 \leq i \leq k-2
$$

Similarly,

$$
R_{i} \geq \chi_{M_{1}}(i), \quad 0 \leq i \leq k-2 .
$$

Now the graph of $g^{(k-2)}$ is a line segment on $[0, t]$ and another line segment on $[t, 1]$ so it has at most two zeros in its support, that is $z_{k-2} \leq 2$. If $k-1 \in M_{0}$, then $g^{(k-1)}(0)=0$ so $g^{(k-2)}$ is constant on $[0, t]$. Whether the constant is zero or non-zero there is no contribution to $z_{k-2}$ from $[0, t]$ so in this case $z_{k-2} \leq 1$. Similarly, if $k-1 \in M_{1}$, then $z_{k-2} \leq 1$. It would violate the Pólya condition (3) for $k-1$ to be in both $M_{0}$ and $M_{1}$ so we may conclude that

$$
2 \geq z_{k-2}+\chi_{M_{0}}(k-1)+\chi_{M_{1}}(k-1) .
$$

Adding the inequalities (8), (9), (10), (11), and (12) and using the fact that

$$
\sum_{i=0}^{k-1}\left(\chi_{M_{0}}(i)+\chi_{M_{1}}(i)\right)=\left|M_{0}\right|+\left|M_{1}\right|=k,
$$

we get the trivial inequality $2 \leq 2$. It follows that the inequalities (8), $(9)$, (10), (11), and (12) are actually equalities. 
Suppose that $S_{0}=[0, t]$. Then $S_{1}=\cdots=S_{k-2}=[0, t]$ and, by continuity, $R_{0}=R_{1}=\cdots=R_{k-2}=1$. Equality in (11) implies that $\{0,1, \ldots, k-2\} \subset$ $M_{1}$. Also, since $S_{k-2}=[0, t]$, the only zero of $g^{(k-2)}$ in $S_{k-2}$ is at $x=t$. Thus $z_{k-2}=1$ and $g^{(k-1)}(0) \neq 0$ so $k-1 \notin M_{0}$. Equality in (12) implies that $k-1 \in M_{1}$ as well and we have $N_{k} \subset M_{1}$ a case which was specifically excluded. We conclude that $S_{0} \neq[0, t]$. Similarly $S_{0} \neq[t, 1]$ so $S_{0}=[0,1]$.

Now equality in (8) implies that $g$ has no zero in the interior of $S_{0}=[0,1]$ which is statement (a).

If $i \in M_{0}$, then as we have noted, standard properties of Green's functions show that $g^{(i)}(0)=0$. On the other hand, if $i \notin M_{0}$, then equality in (10) implies that $L_{i}=0$ and as argued above, $g^{(i)}(0) \neq 0$. This proves statement (b), and (c) follows similarly.

Corollary 2.2 If $i<a$, then $w_{i j}=0$ so $x^{-a} G(x, t)$ is a polynomial when $x \leq t$. Also $(1-x)^{-b} G(x, t)$ is a polynomial when $x \geq t$.

Proof. With $t$ and $g$ as in Proposition 2.1 and $x \leq t$, the formula (4) becomes

$$
g(x)=\sum_{i=0}^{k-1} \sum_{j=0}^{k-1} w_{i j} \frac{x^{i}}{i !} \frac{t^{j}}{j !} .
$$

Proposition 2.1(b) and the definition of $a$ show that $g(0)=g^{\prime}(0)=\cdots=$ $g^{(a-1)}(0)=0$, which is,

$$
\sum_{j=0}^{k-1} w_{i} \frac{t^{j}}{j !}=0 \quad \text { for } i<a .
$$

Since this holds for all $t$ in $(0,1), w_{i j}=0$ for $i<a$ and it is immediate that $x^{-a} G(x, t)$ is a polynomial when $x \leq t$. A similar argument using Proposition 2.1(c) shows that $(1-x)^{-b} G(x, t)$ is a polynomial when $x \geq t$.

For our next application of Proposition 2.1, we introduce the BVP which is adjoint to (2). Let $M_{0}^{*}=\left\{i \in N_{k}: k-1-i \notin M_{0}\right\}$ and $M_{1}^{*}=\left\{i \in N_{k}\right.$ : $\left.k-1-i \notin M_{1}\right\}$. The boundary value problem adjoint to (2) is

$$
v^{(k)}=f ; \quad v^{(i)}(0)=0 \text { for } i \in M_{0}^{*}, \quad v^{(i)}(1)=0 \text { for } i \in M_{1}^{*} .
$$

The transformation $\left(M_{0}, M_{1}\right) \rightarrow\left(M_{0}^{*}, M_{1}^{*}\right)$ is easily visualized in terms of the matrix $E$. To construct the incidence matrix $E^{*}$ for $\left(M_{0}^{*}, M_{2}^{*}\right)$, reverse each row of $E$ and interchange 0's and 1's. So if

$$
E=\left(\begin{array}{cccccc}
1 & 1 & 1 & 0 & 1 & 0 \\
0 & 1 & 0 & 1 & 0 & 0
\end{array}\right) \text {, then } E^{*}=\left(\begin{array}{cccccc}
1 & 0 & 1 & 0 & 0 & 0 \\
1 & 1 & 0 & 1 & 0 & 1
\end{array}\right)
$$


It is easy to see that $\left(M_{0}^{*}, M_{1}^{*}\right)$ satisfies the Pólya condition (3). The numbers $a^{*}, b^{*}, c^{*}$, and $d^{*}$ defined for $\left(M_{0}^{*}, M_{1}^{*}\right)$ as in Definition 1.1 are immediately seen to satisfy $a^{*}=c, b^{*}=d, c^{*}=a$, and $d^{*}=b$. The Green's function for the adjoint BVP is

$$
G^{*}(x, t)=G(t, x)=\frac{(t-x)^{k-1}}{(k-1) !} \chi_{(0, t)}(x)+\sum_{i=0}^{k-1} \sum_{j=0}^{k-1} w_{i j} \frac{t^{i}}{i !} \frac{x^{j}}{j !} .
$$

(See [5].) Applying Proposition 2.1 to the adjoint problem gives the next corollary.

Corollary 2.3 If $j<c$, then $w_{i j}=(-1)^{j+1} \delta_{i+j, k-1}$. (Here $\delta$ is the Kronecker $\delta$-symbol.)

Proof. Let $t$ be in $(0,1)$ and set $g^{*}(x)=G^{*}(x, t)$. If $x<t$ we have

$$
g^{*}(x)=\frac{(t-x)^{k-1}}{(k-1) !}+\sum_{i=0}^{k-1} \sum_{j=0}^{k-1} w_{i} \frac{t^{i}}{i !} \frac{x^{j}}{j !} .
$$

Proposition 2.1(b) and the relation $a^{*}=c$ imply that $g^{*}(0)=g^{* \prime}(0)=\cdots=$ $g^{*(c-1)}(0)=0$, which is,

$$
\frac{(-1)^{j} t^{k-1-j}}{(k-1-j) !}+\sum_{i=0}^{k-1} w_{i} \frac{t^{i}}{i !}=0 \quad \text { for } j<c .
$$

Since this polynomial vanishes for all $t$ in $(0,1)$, all coefficients are zero. That is,

$$
\frac{(-1)^{j}}{i !} \delta_{i+j, k-1}+\frac{w_{i j}}{i !}=0 \text { for } j<c,
$$

which completes the proof.

Corollary 2.4 When $x \leq t, x^{-a}(1-t)^{-d} G(x, t)$ is a polynomial.

Proof. We have already seen that $x^{-a} G(x, t)$ is a polynomial when $x \leq t$ and the last assertion of Corollary 2.2, applied to the adjoint BVP shows that $(1-t)^{-d} G(x, t)$ is also a polynomial when $x \leq t$. The conclusion follows easily.

There is one more ingredient we need before proceeding to estimate the kernel $G(x, t)$. In the next proposition we investigate the behavior of $x^{-a} G(x, t)$ as $x \rightarrow 0$. 
Proposition 2.5 Let $h(t)=\sum_{j=0}^{k-1} w_{a} \frac{t^{j}}{j !}$. Then for $0 \leq i \leq k-1$,

(a) $h(t) \neq 0$ for $t$ in $(0,1)$,

(b) $h^{(i)}(0)=0$ if and only if $i \in M_{0}^{*}$ when $i<k-1-a$,

(c) $h^{(k-1-a)}(0) \neq 0$,

(d) $h^{(i)}(1)=0$ if and only if $i \in M_{1}^{*}$.

Proof. In view of Corollary 2.2 we may define

$$
H(x, t)=x^{-a}\left(G(x, t)-\frac{(x-t)^{k-1}}{(k-1) !} \chi_{(0, x)}(t)\right)=\sum_{i=a}^{k-1} \sum_{j=0}^{k-1} w_{i j} \frac{x^{i-a}}{i !} \frac{t^{j}}{j !}
$$

and note that $h(t)=H(0, t)$.

Since $H(x, t)$ is a polynomial, it is clear that

$$
\begin{aligned}
h^{(i)}(0) & =\lim _{x \rightarrow 0}\left[\left.\left(\frac{\partial}{\partial t}\right)^{i} H(x, t)\right|_{t=0}\right] \\
& =\lim _{x \rightarrow 0}\left[\left.x^{-a}\left(\frac{\partial}{\partial t}\right)^{i} G(x, t)\right|_{t=0}-\frac{(-1)^{i} x^{k-1-i-a}}{(k-1-i) !}\right] .
\end{aligned}
$$

For each fixed $x, G(x, t)$ is a section of the Green's function for the adjoint BVP so if $i \in M_{0}^{*}$, then $\left.\left(\frac{\partial}{\partial t}\right)^{i} G(x, t)\right|_{t=0}=0$. For such an $i$ we have

$$
h^{(i)}(0)=\lim _{x \rightarrow 0}-\frac{(-1)^{i} x^{k-1-i-a}}{(k-1-i) !}=\left\{\begin{array}{ll}
0, & \text { if } i<k-1-a \\
(-1)^{k-a} / a !, & \text { if } i=k-1-a
\end{array} .\right.
$$

This proves (c) and the "if" part of (b).

At the other endpoint we have, if $i \in M_{1}^{*}$,

$$
h^{(i)}(1)=\lim _{x \rightarrow 0}\left[\left.\left(\frac{\partial}{\partial t}\right)^{i} H(x, t)\right|_{t=1}\right]=\lim _{x \rightarrow 0}\left[\left.x^{-a}\left(\frac{\partial}{\partial t}\right)^{i} G(x, t)\right|_{t=1}\right]=0
$$

so the "if" part of $(\mathrm{d})$ holds as well.

Let $T=\left\{i \in M_{0}^{*}: i<k-1-a\right\}$. The definition of $c^{*}$ (which is $a$ ) shows that $k-1-a$ is the largest element of $M_{0}^{*}$. Thus $T$ has exactly one fewer element than $M_{0}^{*}$.

So far we have shown that $h$ satisfies the boundary conditions $h^{(i)}(0)=0$, for $i \in T$, and $h^{(i)}(1)=0$, for $i \in M_{1}^{*}$. The remainder of the proof is similar to the proof of Proposition 2.1. 
Let $z_{i}^{*}$ be the number of zeros of $h^{(i)}$ in its support. Since $h^{(i)}$ is a polynomial, its support is all of $[0,1]$ unless $h^{(i)} \equiv 0$. As we have seen $h^{(k-1-a)}(0) \neq 0$ so $h^{(i)} \not \equiv 0$ for $i \leq k-1-a$.

For $i=0$ we have the obvious inequality

$$
z_{0}^{*} \geq \chi_{T}(0)+\chi_{M_{1}^{*}}(0)
$$

If $h^{(i)} \not \equiv 0$ and $0<i \leq k-1$, then as in Proposition 2.1 we observe that the number of zeros of $h^{(i)}$ in $(0,1)$ is at least $z_{i-1}^{*}-1$ and, adding in the boundary zeros, we have

$$
z_{i}^{*} \geq z_{i-1}^{*}-1+\chi_{T}(i)+\chi_{M_{1}^{*}}(i)
$$

If $h^{(i)} \equiv 0$, then we have $i>k-1-a$ so $i \notin T$. Also, both $z_{i}^{*}$ and $z_{i-1}^{*}$ are zero. It follows that (14) holds in this case as well, reducing to $0 \geq-1+\chi_{M_{1}^{*}}(i)$.

Since the degree of $h$ is at most $k-1$ we must have

$$
0=z_{k-1}^{*}
$$

Adding the inequality (13) and the inequalities (14) for $1 \leq i \leq k-1$ we have

$$
z_{0}^{*}+z_{1}^{*}+\cdots+z_{k-1}^{*} \geq z_{0}^{*}+\cdots+z_{k-2}^{*}-(k-1)+\sum_{i=0}^{k-1}\left(\chi_{T}(i)+\chi_{M_{1}^{*}}(i)\right)
$$

and, applying (15), this simplifies to

$$
0 \geq-(k-1)+\left(|T|+\left|M_{1}^{*}\right|\right)=-(k-1)+(k-1)=0 .
$$

Thus we have equality in (13) and in (14) for $1 \leq i \leq k-1$.

Equality in (13) proves that $h$ has no interior zeros which is (a).

If $h^{(i)} \not \equiv 0$, equality in (14) means that $h^{(i)}$ has no boundary zeros except those accounted for by the terms $\chi_{T}(i)$ and $\chi_{M_{1}^{*}}(i)$. Hence the "only if" parts of (b) and (d) hold when $h^{(i)} \not \equiv 0$.

If $h^{(i)} \equiv 0$, then of course both $h^{(i)}(0)=0$ and $h^{(i)}(1)=0$. As we have seen, $i>k-1-a$ in this case so the "only if" part of (b) holds vacuously. Equality in (14) when $h^{(i)} \equiv 0$ means that $\chi_{M_{1}^{*}}(i)=1$ so $i \in M_{1}^{*}$ and the "only if" part of (d) holds as well.

Corollary 2.6 If $a+c=k$, then $w_{a c-1} \neq 0$ and if $a+c<k$, then $w_{a c} \neq 0$. 
Proof. If $a+c=k$, then Proposition 2.5(c) states that $h^{(c-1)}(0) \neq 0$. This means that $w_{a c-1} \neq 0$.

It is impossible for $a+c=k-1$ so if $a+c<k$ we have $c<k-1-a$. Now by the definition of $a^{*}$ (which is equal to $c$ ) we have $c \notin M_{0}^{*}$ so Proposition 2.5(b) states that $h^{(c)}(0) \neq 0$. This means that $w_{a c} \neq 0$.

We now proceed with the estimate of the Green's function.

Proof of Theorem 1.2. To reduce the number of estimates required we introduce the symmetric BVP. Let $\hat{M}_{0}=M_{1}$ and $\hat{M}_{1}=M_{0}$. This just interchanges the rows of the incidence matrix so it is clear that the Pólya condition (3) still holds. It is easy to see that $\hat{a}=b, \hat{b}=a, \hat{c}=d, \hat{d}=c$, and indeed that $\hat{A}=B, \hat{B}=A, \hat{C}=D$, and $\hat{D}=C$. The Green's function of the symmetric BVP is given by $\hat{G}(x, t)=G(1-x, 1-t)$. (See [5].)

It is enough to prove Theorem 1.2 under the restriction that $x+t \leq 1$ since if (6) and (7) are restricted to $x+t \leq 1$ and applied to the Green's function, $\hat{G}(x, t)$, of the symmetric BVP they become

$$
c_{1}|G(1-x, 1-t)| \leq x^{b}(1-x)^{A} t^{D}(1-t)^{c} \leq c_{2}|G(1-x, 1-t)|,
$$

for $0<x<t \leq 1-x$, and

$$
c_{1}|G(1-x, 1-t)| \leq x^{B}(1-x)^{a} t^{d}(1-t)^{C} \leq c_{2}|G(1-x, 1-t)|,
$$

for $0<t<x \leq 1-t$. Replacing $x$ by $1-x$ and $t$ by $1-t$ we recover $(7)$ and (6) for the original Green's function $G(x, t)$ but restricted to $x+t \geq 1$.

A further reduction shows that it is enough to prove statement (6) since (7) is just (6) applied to the adjoint BVP.

So to complete the proof we must establish (6) under the restriction $x+t \leq$ 1. To do this, it is enough to prove the following five statements.

S1: $x^{-a}(1-x)^{-B} t^{-C}(1-t)^{-d}|G(x, t)|$ is continuous when $0 \leq x \leq t \leq$ $1-x$ except possibly at $(x, t)=(0,0)$. (Strictly speaking we mean that this function, defined on the open set, extends continuously to the closed set with perhaps one exceptional boundary point.)

S2: $0<x^{-a}(1-x)^{-B} t^{-C}(1-t)^{-d}|G(x, t)|<\infty$ for $0<x \leq t \leq 1-x$.

S3: $0<\lim _{x \rightarrow 0} x^{-a}(1-x)^{-B} t^{-C}(1-t)^{-d}|G(x, t)|<\infty$ for $0<t<1$.

S4: $0<\lim _{(x, t) \rightarrow(0,1)} x^{-a}(1-x)^{-B} t^{-C}(1-t)^{-d}|G(x, t)|<\infty$.

S5: $x^{-a}(1-x)^{-B} t^{-C}(1-t)^{-d}|G(x, t)|$ is bounded above and below (away from 0$)$ in some neighborhood of $(0,0)$ when $0<x \leq t$.

On the set $0 \leq x \leq t \leq 1-x$, Corollary 2.4 shows that $x^{-a}(1-t)^{-d} G(x, t)$ is a polynomial, and the function $(1-x)^{-B} t^{-C}$ is continuous on this set except at $(0,0)$. The first statement follows. Proposition 2.1(a) proves S2 
and Proposition 2.5(a) proves S3. Proposition 2.5(d) and the definition of $b^{*}$ (which is $d$ ) imply that

$$
h(1)=h^{\prime}(1)=\cdots=h^{(d-1)}(1)=0 \quad \text { and } \quad h^{(d)}(1) \neq 0
$$

so $(1-t)^{-d} h(t)$ is a polynomial in the variable $1-t$ with a non-zero constant term. Thus, for $x \leq t, x^{-a}(1-t)^{-d} G(x, t)$ is a polynomial in the variables $x$ and $1-t$ with a non-zero constant term. This proves $\mathrm{S} 4$.

We prove S5 in two cases. First suppose that $a+c<k$. In this case $C=c$ so it suffices to prove that $x^{-a} t^{-c} G(x, t)$ has a non-zero limit as $(x, t) \rightarrow 0$ with $0<x \leq t$. By Corollary 2.6, $w_{a c} /(a ! c !)$ is non-zero. We estimate as follows:

$$
\begin{aligned}
& \left|\frac{G(x, t)}{x^{a} t^{c}}-\frac{w_{a c}}{a ! c !}\right|=\left|\left(\sum_{i=a}^{k-1} \sum_{j=0}^{k-1} w_{i j} \frac{x^{i-a}}{i !} \frac{t^{j-c}}{j !}\right)-\frac{w_{a c}}{a ! c !}\right| \\
& \leq \sum_{i=a}^{k-1} \sum_{j=0}^{c-1}\left|w_{i j}\right| \frac{x^{i-a}}{i !} \frac{t^{j-c}}{j !}+\sum_{i=a+1}^{k-1}\left|w_{i c}\right| \frac{x^{i-a}}{i ! c !}+\sum_{i=a}^{k-1} \sum_{j=c+1}^{k-1}\left|w_{i j}\right| \frac{x^{i-a}}{i !} \frac{t^{j-c}}{j !}
\end{aligned}
$$

By Corollary 2.3, $w_{i j}=(-1)^{j+1} \delta_{i+j, k-i}$ for $j<c$ so this becomes

$$
\sum_{i=a}^{k-1} \frac{x^{i-a}}{i !} \frac{t^{k-1-i-c}}{(k-1-i) !}+\sum_{i=a+1}^{k-1}\left|w_{i c}\right| \frac{x^{i-a}}{i ! c !}+\sum_{i=a}^{k-1} \sum_{j=c+1}^{k-1}\left|w_{i j}\right| \frac{x^{i-a}}{i !} \frac{t^{j-c}}{j !}
$$

Now, $0<x \leq t$ and $i-a \geq 0$ so in the first term, $x^{i-a} t^{k-1-i-c} \leq t^{k-1-a-c} \leq t$. The last inequality is justified because $0<t<1$ and $k-1-a-c \geq 1$. (Recall that $a+c=k-1$ is impossible.) In the second term $x^{i-a} \leq x \leq t$ since $i \geq a+1$. In the third term we have $i-a \geq 0$ and $j \geq c+1$ so $x^{i-a} t^{j-c} \leq t$ as well. Thus the entire sum is dominated by a constant multiple of $t$ and so it tends to 0 as $t$ does. This completes the case $a+c<k$.

In the second case, $a+c=k$, we do not have continuity at $(0,0)$ in general so the argument is more delicate. We note that $a>0$ since otherwise $\left(M_{0}, M_{1}\right)$ would satisfy (5) which is prohibited. Recall that in this case $C=c-1$.

$$
\begin{aligned}
x^{-a} t^{-C} G(x, t)=\sum_{i=a}^{k-1} & \sum_{j=0}^{k-1} w_{i j} \frac{x^{i-a}}{i !} \frac{t^{j-c+1}}{j !} \\
& =\sum_{i=a}^{k-1}(-1)^{k-i} \frac{x^{i-a}}{i !} \frac{t^{k-i-c}}{(k-1-i) !}+\sum_{i=a}^{k-1} \sum_{j=c}^{k-1} w_{i j} \frac{x^{i-a}}{i !} \frac{t^{j-c+1}}{j !}
\end{aligned}
$$


where we have used Corollary 2.3 again to simplify $w_{i j}$ for $j<c$. The second term goes to zero with $t$ since $x^{i-a} t^{j-c+1} \leq t$ for each $i$ and $j$. It remains to show that the first term is bounded above and below in absolute value as $(x, t) \rightarrow(0,0)$.

Writing $x$ as $s t$ for some $s \in[0,1]$ and using the hypothesis $a+c=k$, the first term simplifies to

$$
\sum_{i=a}^{k-1}(-1)^{k-i} \frac{s^{i-a}}{i !(k-1-i) !}
$$

which is continuous in $s$ and hence bounded above in absolute value on $[0,1]$. To show that it is also bounded below in absolute value it is enough to show that it has no zero in $[0,1]$. At $s=0$ the value is $(-1)^{k-a} /(a !(k-1-a) !) \neq 0$ so we may complete the argument by recognizing (16) as $(-1)^{k-a} s^{k-1-a} r_{k-1}(s)$ where $r_{n}$ is the function defined in the following lemma.

Lemma 2.7 Suppose $a>0$ and $0<s \leq 1$. For all $n \geq a$,

$$
r_{n}(s)=(-1)^{n-a} \sum_{i=a}^{n} \frac{(-s)^{i-n}}{i !(n-i) !}>0 .
$$

Proof. For $n=a$ we have $r_{a}(s)=1 / a !>0$. Suppose $r_{n}(s)>0$ when $0<s \leq 1$ for some $n \geq a$. Then $r_{n+1}^{\prime}(s)=-r_{n}(s) / s^{2}<0$ so, for $0<s \leq 1$,

$$
r_{n+1}(s) \geq r_{n+1}(1)=\frac{(-1)^{n-a}}{n !} \sum_{i=a}^{n}\left(\begin{array}{c}
n \\
n-i
\end{array}\right)(-1)^{n-i}=\frac{1}{n !}\left(\begin{array}{c}
n-1 \\
n-a
\end{array}\right)>0 .
$$

The last equality is an identity for binomial coefficients. See, for example, [13, p16].

The author would like to thank Alois Kufner for the personal introduction to this problem received during the latter's visit to the University of Western Ontario in May of 1995.

\section{References}

[1] J. S. Bradley,Hardy inequalities with mixed norms, Canad. Math. Bull., 21 (1978), 405-408.

[2] R. H. Cole, Theory of Ordinary Differential Equations, AppletonCentury-Crofts, New York, 1968. 
[3] P. Drábek and A. Kufner, The Hardy inequality and Birkoff interpolation, Bayreuth. -Math. -Schrift., 47 (1994), 99-104.

[4] A. Kufner, A property of Green's function, Real Analysis Exch. 21 (199596), 380-381.

[5] A. Kufner, Higher order Hardy inequalities, Bayreuth. -Math. -Schrift., 44 (1993), 105-146.

[6] A. Kufner and H. P. Heinig, Hardy's inequality for higher order derivatives, Trudy Mat. Inst. Steklov, 192 (1990), 105-113, In Russian, English translation in Proceedings of the Steklov Institute of Mathematics, 3 (1993).

[7] A. Kufner and A. Wannebo, Some remarks on the Hardy inequality for higher order derivatives, General Inequalities VI, 103 of Internat. Series of Numer. Math., pages 33-48. Birkhäuser Verlag, Basel, 1992.

[8] V. G. Maz'ja, Sobolev Spaces, Springer-Verlag, Berlin, Heidelberg, 1985.

[9] B. Muckenhoupt, Hardy's inequality with weights, Studia Math., 44 (1972), 31-38.

[10] B. Opic and A. Kufner, Hardy-type Inequalities, Longman Scientific \& Technical, Longman House, Burnt Mill, Harlow, Essex, England, 1990.

[11] G. Sinnamon, Weighted Hardy and Opial-type inequalities, J. Math. Anal. Appl., 160 (1991), 434-445.

[12] V. D. Stepanov, Weighted inequalities for a class of Volterra convolution operators, J. London Math. Soc., 45 (1992), 232-242.

[13] A. M. Yaglom and I. M. Yaglom, Challenging Mathematical Problems with Elementary Solutions, volume 1, Holden-Day, Inc., San Francisco, 1964, English translation of Neelementarnye zadachi v elementarnom izlozhenii, Government Printing House for Technical-Theoretical Literature, Moscow, 1954. 